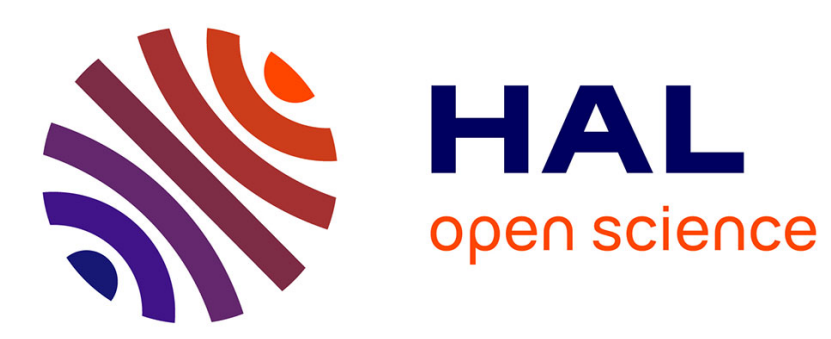

\title{
Analytical models for the inflation of a polymeric tube
}

Fabrice Schmidt, A. Rodriguez-Villa, Jean-François Agassant, Michel Bellet

\section{To cite this version:}

Fabrice Schmidt, A. Rodriguez-Villa, Jean-François Agassant, Michel Bellet. Analytical models for the inflation of a polymeric tube. European Journal of Mechanics - A/Solids, 2000, 19 (1), pp.89-104. 10.1016/S0997-7538(00)00150-9 . hal-02051036

\section{HAL Id: hal-02051036 https://imt-mines-albi.hal.science/hal-02051036}

Submitted on 27 Feb 2019

HAL is a multi-disciplinary open access archive for the deposit and dissemination of scientific research documents, whether they are published or not. The documents may come from teaching and research institutions in France or abroad, or from public or private research centers.
L'archive ouverte pluridisciplinaire HAL, est destinée au dépôt et à la diffusion de documents scientifiques de niveau recherche, publiés ou non, émanant des établissements d'enseignement et de recherche français ou étrangers, des laboratoires publics ou privés. 


\title{
Analytical models for the inflation of a polymeric tube
}

\author{
F.M. Schmidt ${ }^{\mathrm{a}, *}$, A. Rodriguez-Villa $^{\mathrm{b}}$, F. Agassant $^{\mathrm{b}}$, M. Bellet $^{\mathrm{b}}$ \\ ${ }^{a}$ Ecole des mines d'Albi-Carmaux, Campus Jarland, Route de Teillet, 81013 Albi CT Cedex 09, France \\ ${ }^{\mathrm{b}}$ Ecole des mines de Paris, CEMEF-UMR CNRS $n^{\circ}$ 7635, 06904 Sophia-Antipolis, France
}

(Received 10 August 1998; revised 10 August 1999)

\begin{abstract}
In the blow moulding processes, a tube-shaped parison (also called preform) made of polymer is inflated inside a mould in order to obtain the desired bottle shape. The free inflation of a cylindrical tube (a simplified kinematic of inflation for the preform before contacting the mould) has been investigated in order to develop analytical models and then provide reliable validation material for finite element software. The solutions calculated for a tube made of a Newtonian fluid and a Lodge's rubberlike liquid material using both exact volumic approach and thin shell approximation have been analysed and compared.
\end{abstract}

Keywords : elongation flows / tube free inflation / viscoelastic fluid / volumic approach / thin shell assumption / thickness-averaged problem / analytical problem / analytical model / blow moulding process

\section{Introduction}

Elongation flows occur in many industrial processing operations and particularly in the blow moulding process of Polethylene bottles (Rosato, 1989). In a previous paper (Schmidt et al., 1996), numerical finite element simulations of this process were presented using various constitutive equation. The capability of setting up analytical models for the inflation of a tube allows us to validate finite element simulations, to analyse the influence of processing and rheological parameters, and to compare the solutions calculated using a volumic approach and a thin shell assumption.

In the literature, a few works refer to the development of analytical models for polymeric tube free inflation. Chung and Stevenson (1975) have investigated the simultaneous inflation and extension of a cylindrical tube made of a Lodge's rubberlike liquid material (Lodge, 1964) as a method for conducting elongational flow experiments. In this approach, the analytical development is facilitated because the axial displacement is prescribed so that the elongation rate remains constant. Ryan and Dutta $(1982,1984)$ have developed quasianalytical models of free and confined tube inflation using viscoelastic differential constitutive equations. The resulting non-linear equation is solved using an iterative scheme based on a Runge-Kutta method where the components of the stress tensor are computed using Simpson's first rule. In a very similar approach, Schmidt et al. (1996) have developed a simultaneous extension and inflation model of a tube for a Maxwell constitutive equation. Note that in all the previous approaches, the contribution of inertia effects is assumed much smaller than those of viscous and elastic effects. This assumption is justified in the case of elongational flows. Now, if inertia effects are strongly dominant (which is not verified for polymers), Khayat et al. (1992) have found a simple analytical solution for the inner radius.

\footnotetext{
* Corresponding author.
} 
In the following sections, we will present the equations to be solved using both a thin shell approximation and an exact volumic formulation. Applications to Newtonian and Lodge's viscoelastic constitutive equations will be carried out.

\section{Kinematic assumptions}

The radial inflation of a tube (inner radius $R(t)$ ), thickness $e(t)$, constant length $L_{0}$ is considered (figure 1 ). A differential inflation pressure $\Delta P$ is applied to the inner surface of the tube (i.e., at $r=R(t)$ ) and the upper and lower ends of the tube are assumed to move within two fixed horizontal planes (perfectly sliding contact conditions). Hence, the upper and the lower plane can be seen as symmetry planes. The Cauchy stress tensor $\underline{\underline{\sigma}}$ is diagonal at any point of the tube and during all the inflation process. The geometry and boundary conditions being axisymmetric, the following conditions apply to the components of the velocity field $\mathbf{u}$ :

$$
\mathbf{u}=\left(u_{r}(r, t), u_{\theta}=0, u_{Z}=0\right),
$$

$$
\frac{\partial u_{r}}{\partial \theta}=0 .
$$

\section{Mass balance and strain rate tensor}

The material is assumed to be incompressible. Using Eqs (1) and (2), the continuity equations reduces to:

$$
\frac{\partial\left(r u_{r}\right)}{\partial r}=0
$$

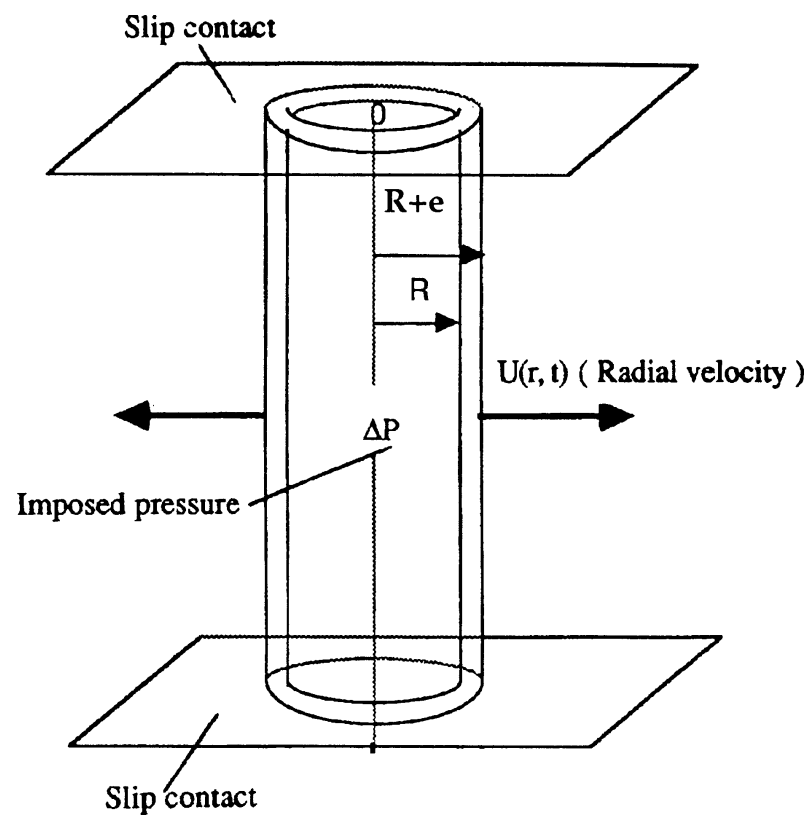

Figure 1. Free inflation of a cylindrical tube. 
Integrating this first-order differential equation, we get:

$$
u_{r}=\frac{A(t)}{r}
$$

where $A$ only depends on time ( $A$ is uniform throughout the tube thickness at each time step). Introducing $u_{r}=\mathrm{d} R / \mathrm{d} t$ at $r=R$, we get:

$$
A(t)=R \frac{\mathrm{d} R}{\mathrm{~d} t}
$$

and thus:

$$
u_{r}=\frac{R}{r} \frac{\mathrm{d} R}{\mathrm{~d} t}
$$

The rate of strain tensor is:

$$
\underline{\underline{\varepsilon}}=\left(\begin{array}{ccc}
-\frac{R}{r^{2}} \frac{\mathrm{d} R}{\mathrm{~d} t} & 0 & 0 \\
0 & \frac{R}{r^{2}} \frac{\mathrm{d} R}{\mathrm{~d} t} & 0 \\
0 & 0 & 0
\end{array}\right) .
$$

Now, if Eq. (3) is integrated along $r$ between the inner radius $R$ and the outer radius $(R+e)$, we obtain:

$$
\int_{R}^{R+e} \frac{\partial\left(r u_{r}\right)}{\partial r} \mathrm{~d} r=0 \Leftrightarrow(R+e) \frac{\mathrm{d}(R+e)}{\mathrm{d} t}-R \frac{\mathrm{d} R}{\mathrm{~d} t}=0 .
$$

Further time integration of this equation from $t^{\prime}=0$ to $t^{\prime}=t$ yields the global volume conservation of the tube:

$$
\pi L_{0}\left((R+e)^{2}-R^{2}\right)=\pi L_{0}\left(\left(R_{0}+e_{0}\right)^{2}-R_{0}^{2}\right),
$$

where $R_{0}$ and $e_{0}$ are, respectively, the initial radius and thickness of the tube. The global volume conservation can be transformed into a more convenient shape using the average radius $\bar{R}=R+(e / 2)$ of the tube:

$$
2 \pi \bar{R} e L_{0}=2 \pi \bar{R}_{0} e_{0} L_{0}
$$

\section{Dynamic equilibrium equations}

In a previous paper (Schmidt et al., 1996), it has been shown that inertia effects remain much lower than viscous and elastic effects in the case of a biaxial deformation of a polymer tube. Thus, the stress balance equations reduce to:

$$
\frac{\partial \sigma_{r r}}{\partial r}+\frac{\sigma_{r r}-\sigma_{\theta \theta}}{r}=0
$$

The stress balance equation in the $r$-direction (Eq. (11)) may be rewritten as follows:

$$
\frac{1}{r} \frac{\partial\left(r \sigma_{r r}\right)}{\partial r}=\frac{\sigma_{\theta \theta}}{r} \Leftrightarrow \frac{\partial\left(r \sigma_{r r}\right)}{\partial r}=\sigma_{\theta \theta}
$$


The imposed pressure yields the following boundary conditions:

$$
\begin{gathered}
r=R: \sigma_{r r}=-\Delta P, \\
r=R+e: \sigma_{r r}=0 .
\end{gathered}
$$

Integrating Eq. (12) from inner to outer radius with boundary conditions (13) and (14) leads to:

$$
\int_{R}^{R+e} \sigma_{\theta \theta} \mathrm{d} r=\Delta P R .
$$

Let us define the thickness averaged orthoradial stress $\bar{\sigma}_{\theta \theta}=(1 / e) \int_{R}^{R+e} \sigma_{\theta \theta} \mathrm{d} r$. Equation (15) can then be rewritten:

$$
\bar{\sigma}_{\theta \theta} e=\Delta P R \Leftrightarrow \bar{\sigma}_{\theta \theta}=\frac{\Delta P R}{e}
$$

which is the average orthoradial stress in the tube independent on the constitutive equation. If the average radius $\bar{R}$ is used:

$$
\bar{\sigma}_{\theta \theta}=\frac{\Delta P \bar{R}}{e}-\frac{\Delta P}{2}
$$

\section{Newtonian behaviour}

Introducing the Newtonian constitutive equation leads to:

$$
\underline{\underline{\varepsilon}}=\left(\begin{array}{ccc}
-p-2 \eta \frac{A}{r^{2}} & 0 & 0 \\
0 & -p+2 \eta \frac{A}{r^{2}} & 0 \\
0 & 0 & -p
\end{array}\right),
$$

where $p$ is the hydrostatic pressure and $\eta$ the viscosity of the material. The stress balance equation leads to a constant hydrostatic pressure at each time step. Using the boundary conditions (13) and (14), we can express $p$ and $A$ as follows:

$$
\left\{\begin{array}{l}
A=\frac{\Delta P(R+e)^{2} R^{2}}{2 \eta\left((R+e)^{2}-R^{2}\right)}, \\
p=-\frac{\Delta P R^{2}}{\left((R+e)^{2}-R^{2}\right)} .
\end{array}\right.
$$

It is to note that $A$ do not depend of $\mathrm{d} R / \mathrm{d} t$ (Eq. (5)). The stress components are:

$$
\left\{\begin{aligned}
\sigma_{r r} & =\frac{\Delta P(R+e)^{2} R^{2}}{\left((R+e)^{2}-R^{2}\right)}\left(\frac{1}{(R+e)^{2}}-\frac{1}{R^{2}}\right), \\
\sigma_{\theta \theta} & =\frac{\Delta P R^{2}}{\left((R+e)^{2}-R^{2}\right)}\left(\frac{1}{(R+e)^{2}}+\frac{1}{R^{2}}\right) \\
\sigma_{z z} & =\frac{\Delta P R^{2}}{\left((R+e)^{2}-R^{2}\right)}
\end{aligned}\right.
$$


Integrating Eq. (11) along $r$ using Eq. (21) and boundary conditions (13) and (14), we find

$$
\Delta P+2 \eta \int_{R}^{R+e}\left(\frac{\partial u_{r}}{\partial r}-\frac{u_{r}}{r}\right) \frac{\mathrm{d} r}{r}=0 \Leftrightarrow \frac{\Delta P}{2 \eta}+\int_{R}^{R+e} \frac{\partial}{\partial r}\left(\frac{u_{r}}{r}\right) \mathrm{d} r=0 .
$$

Introducing $u_{r}=\mathrm{d} r / \mathrm{d} t$, Eq. (21) becomes:

$$
\frac{\Delta P}{2 \eta}+\frac{\mathrm{d}}{\mathrm{d} t}\left(\ln \left(\frac{R+e}{R}\right)\right)=0
$$

And finally:

$$
\left(\frac{R+e}{R}\right)^{2}=\left(\frac{R_{0}+e_{0}}{R_{0}}\right)^{2} \mathrm{e}^{\left(-\frac{\Delta P}{\eta} t\right)} .
$$

Using the volume balance (Eq. (9)) and the initial shape factor $\delta_{0}=e_{0} / R_{0}$, the inner radius $R$ is determined from Eq. (23):

$$
\frac{R}{R_{0}}=\sqrt{\frac{\left(1+\delta_{0}\right)^{2}-1}{\left(1+\delta_{0}\right)^{2} \exp \left(-\frac{\Delta P}{\eta} t\right)-1}} .
$$

The denominator has to remain positive:

$$
\left(1+\delta_{0}\right)^{2} \mathrm{e}^{\left(-\frac{\Delta P}{\eta} t\right)}>1 \Leftrightarrow t<t_{1},
$$

$t_{1}=2 t_{c} \ln \left(1+\delta_{0}\right)$ is the maximum blowing time which depends on a characteristic blowing time $t_{c}=\eta / \Delta P$ and on the initial shape factor $\delta_{0}$. Introducing Eq. (25) into Eq. (24) leads to:

$$
\frac{R}{R_{0}}=\sqrt{\frac{\left(1+\delta_{0}\right)^{2}-1}{\left(1+\delta_{0}\right)^{2\left(1-t / t_{1}\right)}-1}} \quad \forall t \in\left[0, t_{1}[,\right.
$$

which gives the evolution of the inner radius $R$ versus time, for a Newtonian tube.

\section{Viscoelastic behaviour}

The simplest constitutive model of integral type which accounts for both viscous and elastic phenomena is Lodge's model, which is mathematically equivalent to Maxwell's differential model which will be used further on. This model is suitable for large deformations (Agasant et al., 1991). It is written

$$
\underline{\underline{\sigma}}=-q \underline{\underline{I}}+\int_{-\infty}^{t} \frac{\eta}{\lambda^{2}} \mathrm{e}^{-\frac{t-t^{\prime}}{\lambda}} \underline{\underline{C}}^{-1}\left(t^{\prime}\right) \mathrm{d} t^{\prime}
$$

where $q$ is an arbitrary pressure; $\lambda$ is a constant relaxation time and $\underline{\underline{C}}^{-1}\left(t^{\prime}\right)$ is the Finger strain tensor which accounts for large deformation (see Eq. (31)). One can take advantage of the axisymmetric tube growth and use the Lagrangian coordinate transformation $r \rightarrow X$ :

$$
X=\pi\left(r^{2}-R^{2}\right) .
$$


From Eq. (6) it can be deduced that $X$ is a constant for each material point during the inflation process $(\mathrm{d} X / \mathrm{d} t=0)$. When $r$ is equated to the outer radius, $R+e, X$ is equal to the surface of the section of the tube, whereas $X=0$ for the material points located at the inner radius $R$. We have the following relationships:

$$
\begin{aligned}
& \forall t^{\prime} \in[0, t] \quad r^{\prime}=\sqrt{r^{2}-R^{2}+R^{\prime 2}}, \\
& \forall t \leqslant 0 \quad r^{\prime}=r_{0}, R^{\prime}=R_{0} .
\end{aligned}
$$

The components of the gradient tensor $\underline{\underline{F}}$ are:

$$
\underline{\underline{F}}\left(t^{\prime}\right)=\left(\begin{array}{ccc}
\frac{r}{r^{\prime}} & 0 & 0 \\
0 & \frac{r^{\prime}}{r} & 0 \\
0 & 0 & 1
\end{array}\right)
$$

and so we have:

$$
\underline{\underline{C}}^{-1}=\underline{\underline{F}}^{-1} \cdot{ }^{T} \underline{\underline{F}}^{-1}=\left(\begin{array}{ccc}
\left(\frac{r^{\prime}}{r}\right)^{2} & 0 & 0 \\
0 & \left(\frac{r}{r^{\prime}}\right)^{2} & 0 \\
0 & 0 & 1
\end{array}\right) .
$$

Finally, using Eqs (28) and (29), we obtain:

$$
\begin{gathered}
t \geqslant t^{\prime} \geqslant 0 \quad \underline{\underline{C}}^{-1}=\left(\begin{array}{ccc}
\frac{X / \pi+R^{\prime 2}}{X / \pi+R^{2}} & 0 & 0 \\
0 & \frac{X / \pi+R^{2}}{X / \pi+R^{\prime 2}} & 0 \\
0 & 0 & 1
\end{array}\right), \\
\forall t \geqslant 0 \text { and } t^{\prime} \leqslant 0 \quad \underline{\underline{C}}^{-1}=\left(\begin{array}{ccc}
\frac{X / \pi+R_{0}^{2}}{X / \pi+R^{2}} & 0 & 0 \\
0 & \frac{X / \pi+R^{2}}{X / \pi+R_{0}^{2}} & 0 \\
0 & 0 & 1
\end{array}\right) .
\end{gathered}
$$

In order to simplify the calculation, dimensionless variables are introduced:

$$
\underline{\underline{\tilde{\sigma}}}=\lambda \stackrel{\underline{\underline{\sigma}}}{\eta}, \quad \tilde{t}=\frac{t}{\lambda}, \quad \widetilde{R}=\frac{R}{R_{0}}, \quad \widetilde{X}=\frac{X}{\pi R_{0}^{2}}, \quad \widetilde{S}=\frac{S}{\pi R_{0}^{2}}=\left(\frac{R_{0}+e_{0}}{R_{0}}\right)^{2}-1,
$$

$\widetilde{S}$ is the dimensionless surface of the transverse section of the tube. Using Eq. (27), the components of $\underline{\underline{\sigma}}$ are:

$$
\begin{gathered}
\tilde{\sigma}_{r r}=-\tilde{q}+\frac{\mathrm{e}^{-\tilde{t}}}{\widetilde{X}+\widetilde{R}^{2}}\left(\widetilde{X}+1+\int_{0}^{\tilde{t}} \mathrm{e}^{\tilde{t}^{\prime}}\left(\widetilde{X}+\widetilde{R}^{\prime 2}\right) \mathrm{d} \tilde{t}^{\prime}\right) \\
\tilde{\sigma}_{\theta \theta}=-\tilde{q}+\mathrm{e}^{-\tilde{t}}\left(\widetilde{X}+\widetilde{R}^{2}\right)\left(\frac{1}{\widetilde{X}+1}+\int_{0}^{\tilde{t}} \frac{\mathrm{e}^{\tilde{t}^{\prime}} \mathrm{d} \tilde{t}^{\prime}}{\widetilde{X}+\widetilde{R}^{\prime 2}}\right),
\end{gathered}
$$




$$
\tilde{\sigma}_{z z}=-\tilde{q}+1
$$

The stress balance equation in the $z$-direction reduces to:

$$
\frac{\partial \tilde{q}}{\partial z}=0 \Leftrightarrow \tilde{q}=\tilde{q}(\tilde{X}, \tilde{t})
$$

In addition, if we integrate the stress balance Eq. (11) using boundary conditions (13), (14) and the previous dimensionless variables, we get:

$$
\operatorname{De}_{v}+\frac{1}{2} \int_{0}^{\bar{\vartheta}} \frac{\bar{\sigma}_{r r}-\bar{\sigma}_{\theta \theta}}{\widetilde{X}^{\prime}+\widetilde{R}^{2}} \mathrm{~d} \widetilde{X}^{\prime}=0
$$

where $\operatorname{De}_{v}=\lambda(\Delta P / \eta)$ is the Deborah number. The non-linear form of Eq. (39) excludes any simple analytical solution for $\widetilde{R}$ except for some limiting cases (see Section 7). A numerical algorithm has thus been developed to solve Eq. (39), details can be found in (Schmidt, 1995). At each time step, the dimensionless radius $\widetilde{R}$ is determined from Eq. (39) using a quasi-Newton iterative procedure. The iterative scheme was stopped when successive values of $\widetilde{R}$ differed by less than $10^{-3}$ percent. Simpson's first rule was used to evaluate the integrals. Then, the components of the stress tensor are deduced.

\section{Thin shell approximation}

In this paragraph we propose an analytical solution for thin tubes, i.e. the thickness of which is much smaller than the radius $\delta_{0}=e_{0} / R_{0} \ll 1$. It is clear that, in that particular geometry, no shear stress develop during the inflation process since the geometry remains axisymmetrical, i.e., the radial normal vector remains normal during inflation (see (18) and (31)). In that context thin shell approximation leads to neglect the radial stress $\sigma_{r r}$, which is $\mathrm{O}(\Delta P)$, in front of the orthoradial stress $\sigma_{\theta \theta}$, which is $\mathrm{O}(\Delta P(R / e))$. Hence, a plane stress state will be considered, and the thickness-averaged problem is solved.

\subsection{Mean strain-rate tensor}

In order to develop a thin shell approach, we average the volumic strain-rate tensor $\underline{\underline{\varepsilon}}$ over the tube's thickness. We define an average strain-rate tensor $\underline{\underline{\dot{\varepsilon}}}$ :

$$
\underline{\underline{\dot{\varepsilon}}}=\frac{1}{e} \int_{R}^{R+e} \quad \underline{\underline{\varepsilon}} \mathrm{d} r
$$

From Eq. (7) we get:

$$
\underline{\underline{\dot{\varepsilon}}}=\left(\begin{array}{ccc}
-\frac{1}{\bar{R}} \frac{\mathrm{d} \bar{R}}{\mathrm{~d} t} & 0 & 0 \\
0 & \frac{1}{\bar{R}} \frac{\mathrm{d} \bar{R}}{\mathrm{~d} t} & 0 \\
0 & 0 & 0
\end{array}\right)
$$




\subsection{Evolution of the tube radius. Maxwell's behaviour}

We introduce the upper convected Maxwell model which is equivalent to the integral Lodge equation (27): It is well known that these equations are suitable for large deformations.

$$
\begin{gathered}
\underline{\underline{\underline{\sigma}}}=-\bar{q} \underline{\underline{I}}+\underline{\underline{\bar{\tau}}}, \\
\underline{\underline{\underline{\tau}}}+\lambda \underline{\underline{\left(\frac{\delta \tau}{\delta t}\right)}}=2 \eta \underline{\underline{\underline{\varepsilon}}}
\end{gathered}
$$

where $\bar{q}$ is the average pseudo-pressure and $\underline{\bar{\tau}}$, the average extra-stress tensor. It should be noted that $\lambda=0$ yields the Newtonian model, $\bar{q}$ then being the hydrostatic pressure and $\underline{\underline{\tau}}$ the deviatoric stress tensor.

Also note that the equation has been written directly with the average tensors. As stress and rate of strain tensors are diagonal, the upper convected derivative of the tensor is:

$$
\underline{\underline{\left(\frac{\delta \tau}{\delta t}\right)}}=\frac{\mathrm{d} \overline{\underline{\underline{\tau}}}}{\mathrm{d} t}-2 \underline{\underline{\dot{\varepsilon} \bar{\tau}}}
$$

We are considering here a plane stress state. Pseudo-pressure and orthoradial stress components are solution of the following differential equations:

$$
\begin{gathered}
\bar{q}+\lambda \frac{\mathrm{d} \bar{q}}{\mathrm{~d} t}-2 \lambda \bar{q} \overline{\dot{\varepsilon}}_{r r}=2 \eta \overline{\dot{\varepsilon}}_{r r}, \\
\bar{\sigma}_{\theta \theta}+\bar{q}+\lambda \frac{\mathrm{d}\left(\bar{\sigma}_{\theta \theta}+\bar{q}\right)}{\mathrm{d} t}-2 \lambda\left(\bar{\sigma}_{\theta \theta}+\bar{q}\right) \overline{\dot{\varepsilon}}_{\theta \theta}=2 \eta \overline{\dot{\varepsilon}}_{\theta \theta} .
\end{gathered}
$$

Introducing average strain-rate tensor $\underset{\underline{\dot{\varepsilon}}}{\underline{E}}$ Eq. (45)), equilibrium equation (Eq. (17)) (in which we use the membrane assumption:

$$
\bar{\delta}_{0}=e_{0} / R_{0} \ll 1 \quad\left(\text { where } \bar{\delta}_{0} /\left(1+\delta_{0} / 2\right) \cong \delta_{0} \text { if } \delta_{0} \ll 1\right)
$$

and mass conservation (Eq. (10)) lead to a system of two differential equations with pseudo-pressure $\bar{q}$ and membrane radius $\bar{R}$ as unknowns. Note that the imposed internal pressure $\Delta P$ has been assumed an arbitrary function of time, and that $\bar{q}$ and $\bar{R}$ are only functions of time

$$
\begin{gathered}
2 \bar{R}_{0} e_{0}\left(\bar{q}+\lambda \frac{\mathrm{d} \bar{q}}{\mathrm{~d} t}\right)=-\left(\Delta P+\lambda \frac{\mathrm{d}(\Delta P)}{\mathrm{d} t}\right) \bar{R}^{2}, \\
4 \bar{R}_{0} e_{0} \frac{\mathrm{d} \bar{R}}{\mathrm{~d} t}(\lambda \bar{q}+\eta)=\left(\Delta P+\lambda \frac{\mathrm{d}(\Delta P)}{\mathrm{d} t}\right) \bar{R}^{3} .
\end{gathered}
$$

Up to our knowledge this system of equations does not have general analytical solutions. Nevertheless, for particular prescribed pressure curves $\Delta P(t)$, these solutions do exist. 


\subsection{Initial condition on the imposed pressure curve}

The above development is valid for any arbitrary imposed pressure $\Delta P=\Delta P(t)$. Extra-stress tensor $\underline{\underline{\underline{\tau}}}$ in the membrane is assumed to be zero before blowing (say time $t=0$ ). Due to the plane stress state approximation, the pseudo-pressure's value at this moment is zero too $\left(\bar{q}(0)=\bar{\tau}_{r r}(0)-\bar{\sigma}_{r r}(0)=0\right)$, and thus the value of orthoradial stress $\bar{\sigma}_{\theta \theta}$ must also be zero $\left(\bar{\sigma}_{\theta \theta}(0)=-\bar{q}(0)+\bar{\tau}_{\theta \theta}(0)\right)$. Equilibrium equation (17) shows that the imposed pressure must then also start from zero:

$$
\Delta P(t=0)=0 .
$$

Note that in the case of a Newtonian behaviour $(\lambda=0)$, it is not necessary to impose a pressure at blowing start up.

\subsection{Abrupt step approximation}

For comparison's sake with the classical problem of a Newtonian membrane inflating under a constant pressure load $\Delta P(t)=P_{0}$, we are led to assume an abrupt increase of pressure from the initial value $\Delta P=0$ to a constant regime value $P_{0}$ (figure 2). Let us suppose that this increase takes place from time $t=-\Delta t$ to time $t=0$, interval $\Delta t$ being arbitrary small, in particular considerably smaller than relaxation time $\lambda$. The imposed pressure is thus:

$$
\begin{gathered}
\Delta P(t)=P_{0}\left(1+\frac{t}{\Delta t}\right) \quad \text { if }-\Delta t \leqslant t \leqslant 0, \\
\Delta P(t)=P_{0} \quad \text { if } t>0 .
\end{gathered}
$$

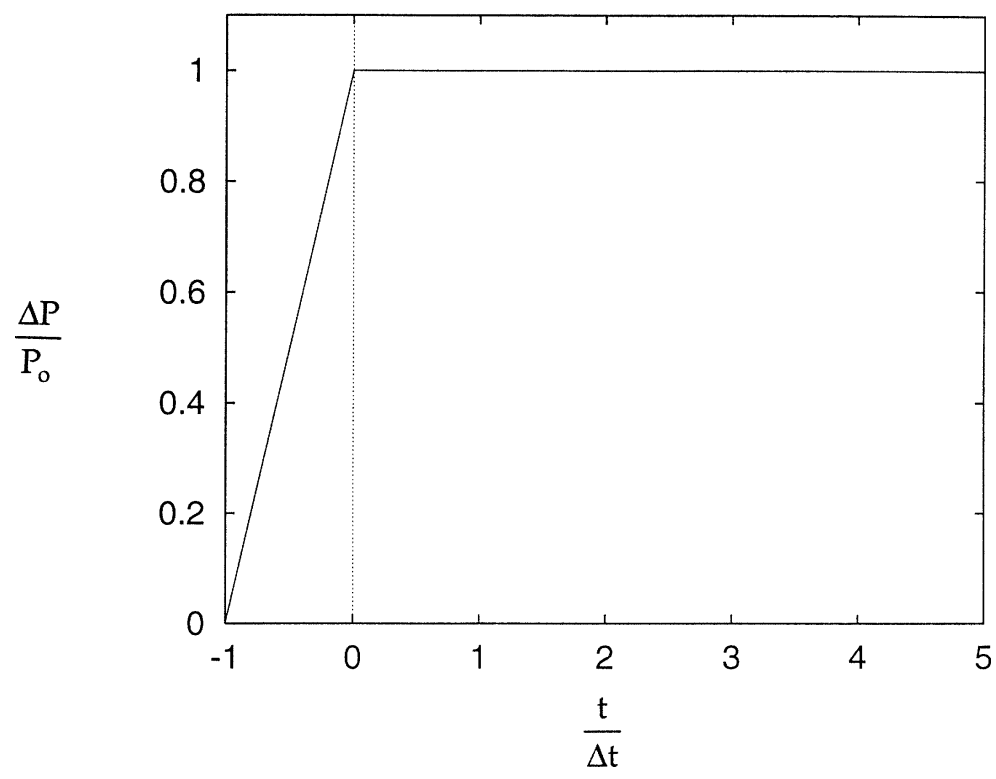

Figure 2. Abrupt increase of pressure load. 


\subsection{Abrupt step solutions}

Let us consider the first "fictitious" part of the pressure curve, from $t=-\Delta t$ to $t=0$. Initial conditions are:

$$
\left\{\begin{array}{l}
\bar{R}(t=-\Delta t)=\bar{R}_{-\Delta t}, \\
e(t=-\Delta t)=e_{-\Delta t}=\frac{\bar{R}_{0} e_{0}}{\bar{R}_{-\Delta t}} \quad \text { by volume conservation, } \\
\bar{q}(t=-\Delta t)=0 .
\end{array}\right.
$$

Introducing Eqs (54), (51) and (52) lead to:

$$
\begin{gathered}
2 \bar{R}_{-\Delta t} e_{-\Delta t}\left(\bar{q}+\lambda \frac{\mathrm{d} \bar{q}}{\mathrm{~d} t}\right)=-P_{0}\left(1+\frac{t}{\Delta t}+\frac{\lambda}{\Delta t}\right) \bar{R}^{2}, \\
4 \bar{R}_{-\Delta t} e_{-\Delta t} \frac{\mathrm{d} \bar{R}}{\mathrm{~d} t}(\lambda \bar{q}+\eta)=P_{0}\left(1+\frac{t}{\Delta t}+\frac{\lambda}{\Delta t}\right) \bar{R}^{3} .
\end{gathered}
$$

The time interval during which this pressure increase takes place has been chosen arbitrary small with respect to the relaxation time $\lambda$ of the material:

$$
\begin{gathered}
\left|\frac{\lambda}{\Delta t}\right| \gg 1, \\
\left|\frac{\lambda}{\Delta t}\right| \gg \frac{t}{\Delta t}=\mathrm{O}(1) .
\end{gathered}
$$

Thus, Eqs (57) and (58), together with volume conservation (Eq. (10)), can be approximated by:

$$
\begin{gathered}
2 \bar{R}_{0} e_{0}\left(\bar{q}+\lambda \frac{\mathrm{d} \bar{q}}{\mathrm{~d} t}\right)=-P_{0} \frac{\lambda}{\Delta t} \bar{R}^{2}, \\
4 \bar{R}_{0} e_{0} \frac{\mathrm{d} \bar{R}}{\mathrm{~d} t}(\lambda \bar{q}+\eta)=P_{0} \frac{\lambda}{\Delta t} \bar{R}^{3} .
\end{gathered}
$$

At time $t=-\Delta t$, taking into account boundary conditions (56), one obtains:

$$
\begin{aligned}
& \left.\frac{\mathrm{d} \bar{q}}{\mathrm{~d} t}\right|_{-\Delta t}=-\frac{P_{0} \bar{R}_{-\Delta t}^{2}}{2 \bar{R}_{0} e_{0} \Delta t}, \\
& \left.\frac{\mathrm{d} \bar{R}}{\mathrm{~d} t}\right|_{-\Delta t}=-\frac{P_{0} \lambda \bar{R}_{-\Delta t}^{3}}{4 \eta \bar{R}_{0} e_{0} \Delta t} .
\end{aligned}
$$

One introduces then a Taylor development of $\bar{q}$ and $\bar{R}$ from $t=-\Delta t$ to $t=0$ :

$$
\begin{gathered}
\bar{q}_{0}=\bar{q}_{-\Delta t}+\left.\frac{\mathrm{d} \bar{q}}{\mathrm{~d} t}\right|_{-\Delta t} \Delta t, \\
\bar{R}_{0}=\bar{R}_{-\Delta t}+\left.\frac{\mathrm{d} \bar{R}}{\mathrm{~d} t}\right|_{-\Delta t} \Delta t,
\end{gathered}
$$


where $\bar{q}_{0}$ is the pseudo-pressure at $t=0$. Introducing Eqs (63) and (64) in this Taylor development leads to an algebraic system of equations, with $\bar{q}_{0}$ and $\bar{R}_{-\Delta t}$ as unknowns:

$$
\begin{gathered}
\bar{q}_{0}=-\frac{\bar{R}_{-\Delta t}^{2}}{A}, \\
\frac{1}{B} \bar{R}_{-\Delta t}^{3}+\bar{R}_{-\Delta t}-\bar{R}_{0}=0,
\end{gathered}
$$

with $A=2 \bar{R}_{0} e_{0} / P_{0}, B=2 \eta A / \lambda$. Constant $B$ is always positive, and Eq. (68) has a single real solution, given by

$$
\bar{R}_{-\Delta t}=\sqrt[3]{\frac{B \bar{R}_{0}}{2}+\sqrt{\frac{B^{2} \bar{R}_{0}^{2}}{4}+\frac{B^{3}}{27}}}+\sqrt[3]{\frac{B \bar{R}_{0}}{2}-\sqrt{\frac{B^{2} \bar{R}_{0}^{2}}{4}+\frac{B^{3}}{27}}} .
$$

The expression of $\bar{q}_{0}$, necessary as an initial condition to the second part of the inflation (constant pressure regime), is then found by replacing this $\bar{R}_{-\Delta t}$ value in Eq. (67)

\subsection{Constant load solutions}

Let us consider the second part of the imposed pressure curve (Eq. (55)). Initial conditions are:

$$
\begin{gathered}
\bar{R}(0)=\bar{R}_{0}, \\
e(0)=e_{0}, \\
\bar{q}(0)=\bar{q}_{0},
\end{gathered}
$$

where $\bar{q}_{0}$ has been calculated in the previous paragraph.

\subsection{Newtonian fluids}

As said earlier, the abrupt step approximation is not required for the Newtonian case (for $\lambda=0$ Eq. (68) leads to $\left.\bar{R}_{-\Delta t}=\bar{R}_{0}\right)$, and thus $\bar{q}_{0}=-P_{0} \bar{R}_{0} /\left(2 e_{0}\right)$. Equation (52) gives the mean radius evolution for a Newtonian tube:

$$
\frac{1}{\bar{R}^{3}} \frac{\mathrm{d} \bar{R}}{\mathrm{~d} t}=\frac{P_{0}}{4 \eta \bar{R}_{0} e_{0}} .
$$

This equation is integrated from $t^{\prime}=0$ to $t^{\prime}=t$ and yields:

$$
\frac{\bar{R}(t)}{\bar{R}_{0}}=\frac{1}{\sqrt{1-\frac{t}{t_{1 N}}}}
$$

where

$$
t_{1 N}=\frac{2 \eta e_{0}}{P_{0} \bar{R}_{0}}
$$


is the Newtonian limit inflating time for a membrane. It is interesting to compare this result with the limit volumic blowing time $t_{1}$ (Eq. (25) of Section 5) when the shape factor $\delta_{0}$ becomes small:

$$
\lim _{\delta_{0} \rightarrow 0} t_{1}=\frac{2 \eta}{P_{0}}\left(\delta_{0}+\mathrm{O}\left(\delta_{0}^{2}\right)\right)=t_{1 N}
$$

\subsection{Viscoelastic fluids}

Let us now consider the more general case of a Maxwell fluid submitted to a constant pressure load. Equations (51), (52) are simplified:

$$
\begin{gathered}
2 \bar{R}_{0} e_{0}\left(\bar{q}+\lambda \frac{\mathrm{d} \bar{q}}{\mathrm{~d} t}\right)=-P_{0} \bar{R}^{2} \\
4 \bar{R}_{0} e_{0} \frac{\mathrm{d} \bar{R}}{\mathrm{~d} t}(\lambda \bar{q}+\eta)=P_{0} \bar{R}^{3}
\end{gathered}
$$

Including

$$
\xi=\lambda \bar{q}+\eta=\frac{P_{0} \bar{R}_{3}}{4 \bar{R}_{0} e_{0} \frac{\mathrm{d} \bar{R}}{\mathrm{~d} t}}
$$

leads to:

$$
\begin{gathered}
\frac{2}{\bar{R}} \frac{\mathrm{d} \bar{R}}{\mathrm{~d} t}=\frac{4 \eta \bar{R}_{0} e_{0}}{\lambda P_{0}} \frac{1}{\bar{R}^{3}} \frac{\mathrm{d} \bar{R}}{\mathrm{~d} t}-\frac{1}{\xi} \frac{\mathrm{d} \xi}{\mathrm{d} t}-\frac{1}{\lambda}, \\
4 \bar{R}_{0} e_{0} \frac{\mathrm{d} \bar{R}}{\mathrm{~d} t} \xi=P_{0} \bar{R}^{3} .
\end{gathered}
$$

Integrating Eq. (80) from $t^{\prime}=0$ to $t^{\prime}=t$, one gets:

$$
\ln \left(\frac{\bar{R}}{\bar{R}_{0}}\right)^{2}=K\left(\frac{1}{\bar{R}_{0}^{2}}-\frac{1}{\bar{R}^{2}}\right)-\ln \left(\frac{\xi}{\xi_{0}}\right)-\frac{t}{\lambda},
$$

where $K=2 \eta \bar{R}_{0} e_{0} /\left(\lambda P_{0}\right)$ and $\xi_{0}=\lambda \bar{q}_{0}+\eta$. Exponentiating Eq. (82) and performing variable separation one gets:

$$
\frac{P_{0}}{\bar{R}_{0} e_{0}} \mathrm{e}^{t / \lambda} \mathrm{d} t=4 J_{0} \bar{R}^{-5} \mathrm{e}^{-K / \bar{R}^{2}} \mathrm{~d} \bar{R}
$$

with $J_{0}=\xi_{0} \bar{R}_{0}^{2} \mathrm{e}^{K / \bar{R}_{0}^{2}}$. Equation (83) can be integrated as a function of time:

$$
\left(1+\frac{K}{\bar{R}^{2}}\right) \mathrm{e}^{-K / \bar{R}^{2}}=\left(1+\frac{K}{\bar{R}_{0}^{2}}\right) \mathrm{e}^{-K / \bar{R}_{0}^{2}}+\frac{K^{2} \lambda P_{0}}{2 \bar{R}_{0} e_{0} J_{0}}\left[\mathrm{e}^{t / \lambda}-1\right] .
$$

This equation does not provide explicit $\bar{R}$ value at a given time step but the blowing time may be calculated explicitly. Equation (84) leads finally to:

$$
\frac{t(\bar{R})}{\lambda}=\ln \left\{1+\frac{\left(\mathrm{De}_{s}+\left(\frac{\bar{R}_{0}}{\bar{R}}\right)^{2}\right) \mathrm{e}^{\left[\frac{1}{\mathrm{De}}\left(1-\left(\frac{\bar{R}_{0}}{\overline{\bar{R}}}\right)^{2}\right)\right]}-\mathrm{De}_{s}-1}{\left.2 \frac{t_{1 N}}{\overline{R_{0}}} \frac{\mathrm{d} \bar{R}}{\mathrm{~d} t}\right|_{0}}\right\}
$$


where $\mathrm{De}_{s}$ is the Deborah number defined here as $\mathrm{De}_{s}=\lambda / t_{1 N}$; we need to known the derivative of $\bar{R}$ at time $t=0$. This value is directly linked to the known value (Eq. (62)):

$$
\left.\frac{\mathrm{d} \bar{R}}{\mathrm{~d} t}\right|_{0}=\frac{\lambda P_{0} \bar{R}_{0}^{2}}{4 e_{0}\left(\lambda \bar{q}_{0}+\eta\right)}
$$

As in the Newtonian case, a limit value of inflation time may be obtained $(\bar{R} \rightarrow+\infty$ in Eq. (85)):

$$
t_{1 M}=\lambda \ln \left\{1+\left.\frac{\mathrm{De}_{s} \mathrm{e}^{1 / \mathrm{De}}-\mathrm{De}_{s}-1}{2 \frac{t_{1 N}}{\bar{R}_{0}} \mathrm{~d} \bar{R} t}\right|_{0}\right\}
$$

\section{Comparison of volumic and thin shell solutions}

We compare the results given by both models. Viscosity is $\eta=50 \mathrm{kPas}$. Prescribed pressure is $10 \mathrm{kPa}$. We have tested three values of $\delta_{0}: \delta_{0}=0.01, \delta_{0}=0.1, \delta_{0}=0.5$, for two different fluids ( $\lambda=0$ (Newtonian), $\lambda=0.01 \mathrm{~s}$ (viscoelastic)). In the Newtonian case, figure 3 shows the evolution of the dimensionless mean radius $\bar{R} / \bar{R}_{0}$ versus time. For $\delta_{0}=0.01$ and $\delta_{0}=0.1$ volumic and thin shell solutions are superimposed; even at $\delta_{0}=0.5$, the difference remains limited.

In the Newtonian case we draw the orthoradial stress distribution across the thickness of the tube, at midinflation, for the same values of $\delta_{0}$ (figure 4). It clearly appears that the difference between volumic and thin shell orthoradial stresses becomes significant even for $\delta_{0}=0.1$. So, in term of kinematic evolution, the thin shell assumption is correct but not in term of stress prediction.

These phenomena are slightly emphasised in the viscoelastic case. Difference between shell and volumic approaches remains of the same order of magnitude for this small value of the Deborah number (figures 5, 6).

It is to note that if we compare the volumic approach with a thin shell solution but using, as this is classically done, the inner radius instead of the mean radius the difference would be more important (Agasant et al., 1996).

The limit viscoelastic blowing time (Eq. (87)) as a function of the Maxwell relaxation time $\lambda$ is presented on figure 7 . When increasing $\lambda$ the limit blowing time decreases and even becomes negative. This may be related to unattainable blowing conditions equivalent to unattainable stretching conditions encountered in fibre spinning and in cast film (Agasant et al., 1996; Rodriquez-Villa, 1997). The unattainable blowing condition become more drastic when the shape factor of the tube $\delta_{0}$ decreases.

\section{Conclusion}

Analytical models for the free inflation of a polymeric tube have been developed for volumic approach and thin shell approximation. They have been compared for Newtonian and Maxwell fluids.

They point out that the thin shell approximation (with an average radius $\bar{R}$ ) remains valid in term of kinematic evolution up to high values of the initial shape factor $\left(\delta_{0}=0.5\right)$. On the other hand, the difference between volumic and thin shell orthoradial stresses becomes significant for lower values of the initial shape factor. Stresses are significantly underestimated when using a thin shell approximation. It is to notice that more sophisticated finite strain shell theories should reduce these discrepancies.

A limit blowing time may be derived in the Newtonian case for both volumic case and thin shell approaches. The asymptotic value is the same when the initial tube shape factor tends to zero. 


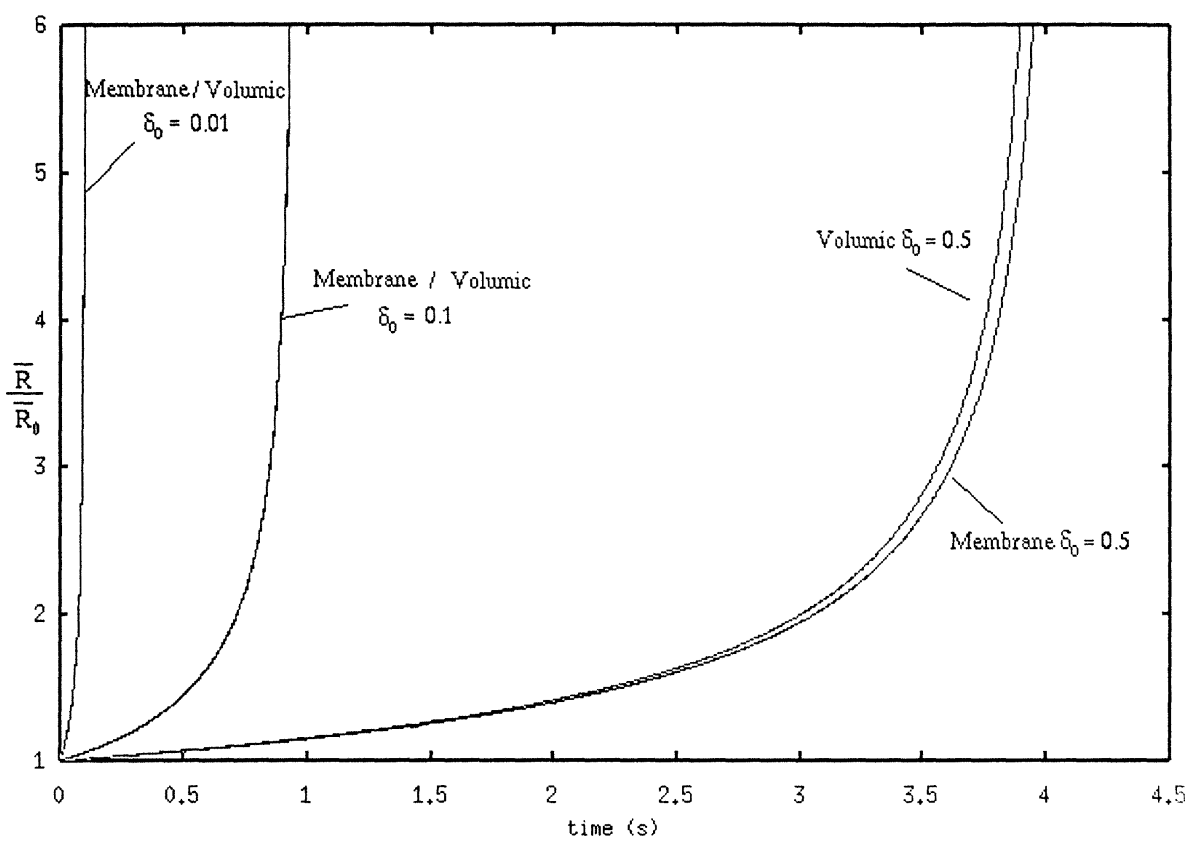

Figure 3. Comparison of radii evolutions for a Newtonian fluid.

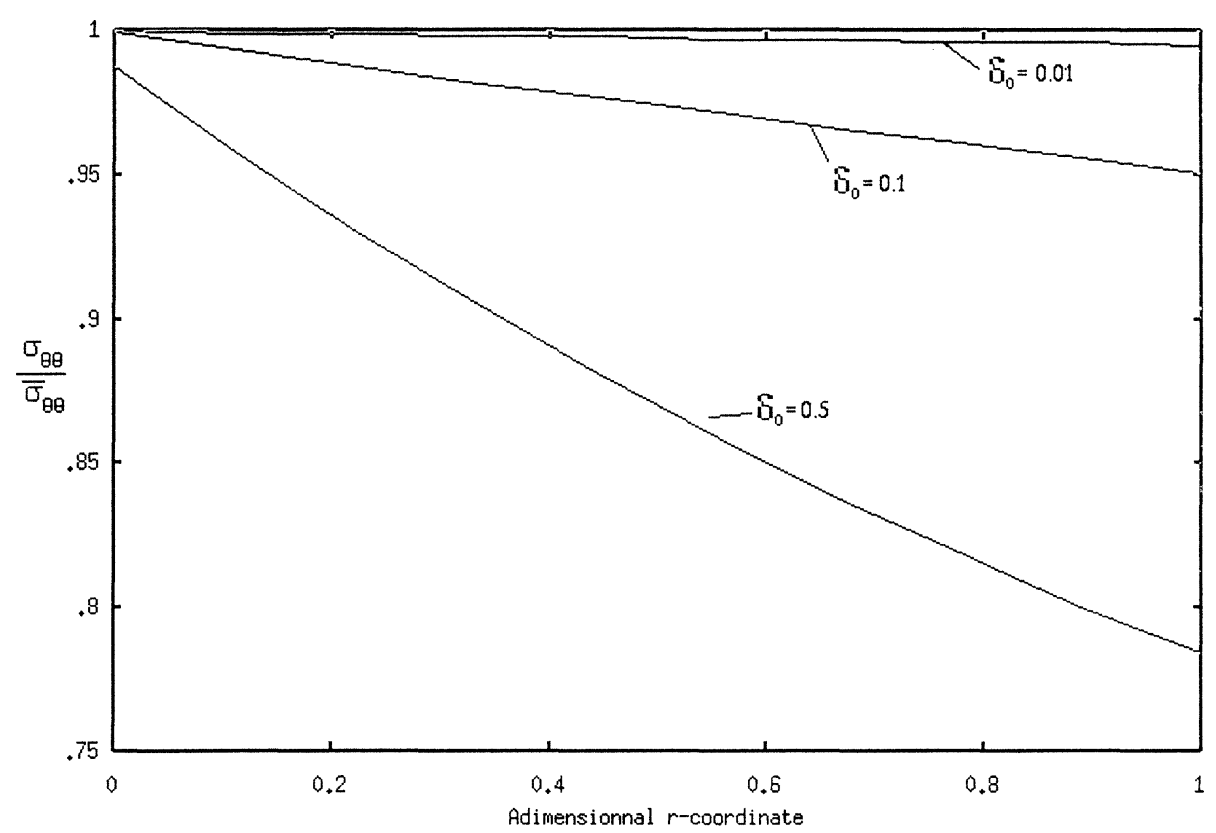

Figure 4. Comparison of orthoradial stress distribution across the tube thickness at mid-inflation (Newtonian).

In the viscoelastic case, a limit viscoelastic blowing time may be derived when using the thin shell approximation. This limit blowing time decreases with the material characteristic relaxation time and may becomes negative for values of the relaxation time exceeding a critical value, which is physically impossible. This could be related to unattainable blowing conditions which are also encountered in other stretching processing (fibre spinning, film drawing). 


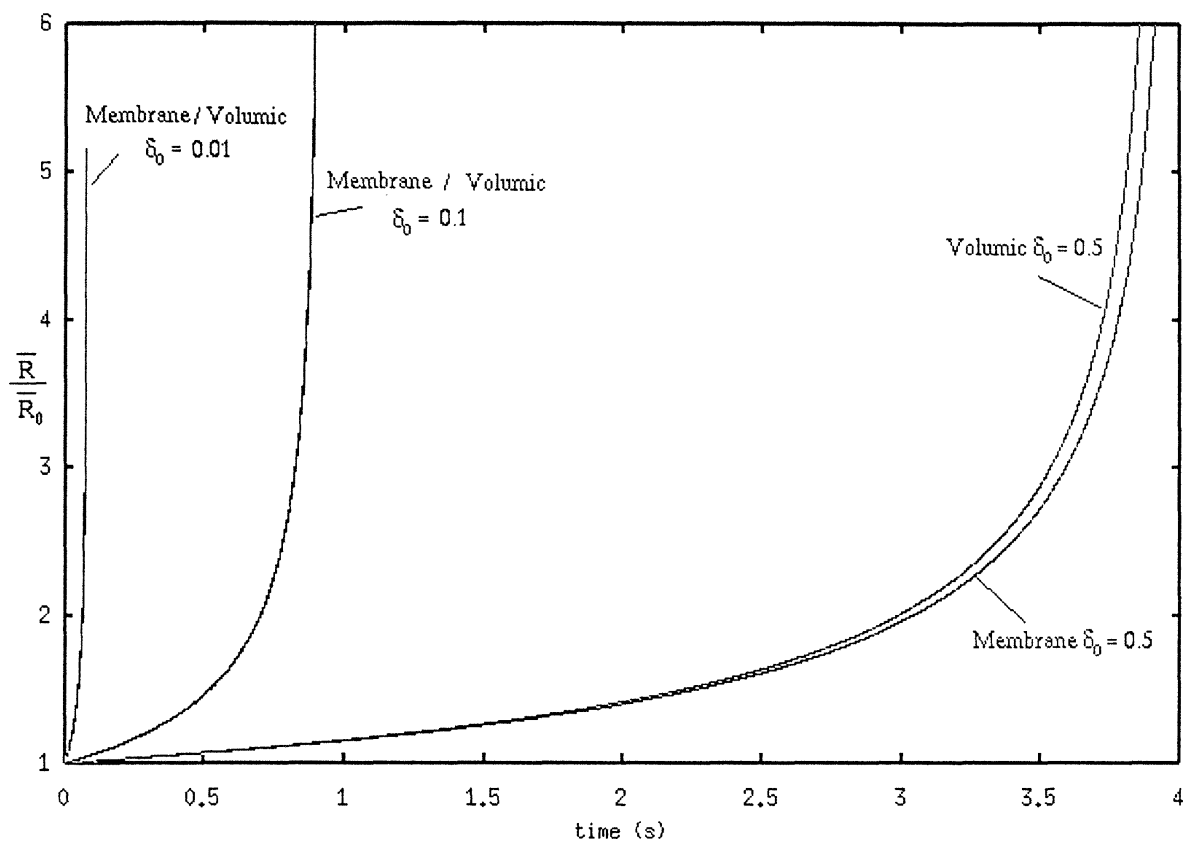

Figure 5. Comparison of radii evolutions for a viscoelastic fluid $\lambda=0.01 \mathrm{~s}$.

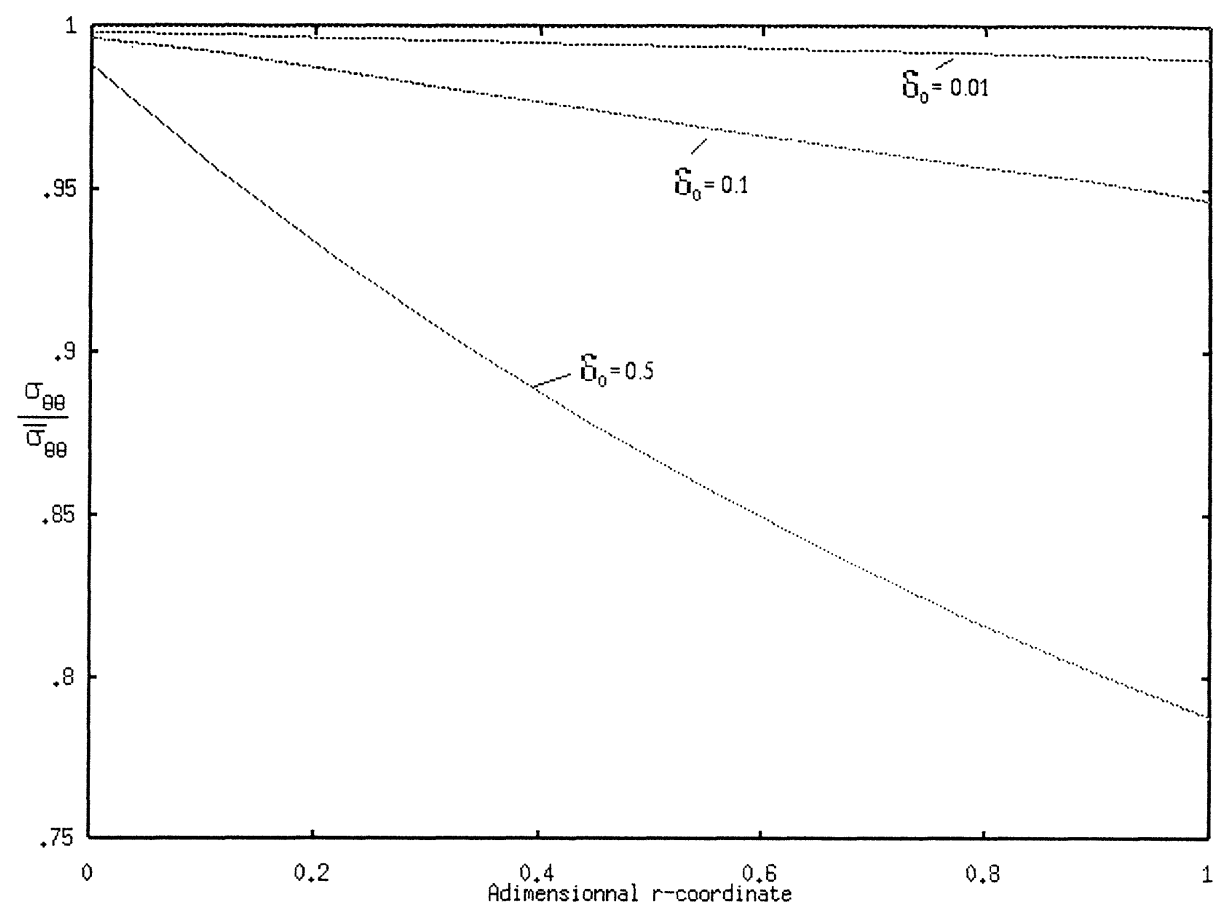

Figure 6. Comparison of orthoradial stress distribution across the tube thickness at mid-inflation (viscoelastic fluid $\lambda=0.01 \mathrm{~s}$ ). 


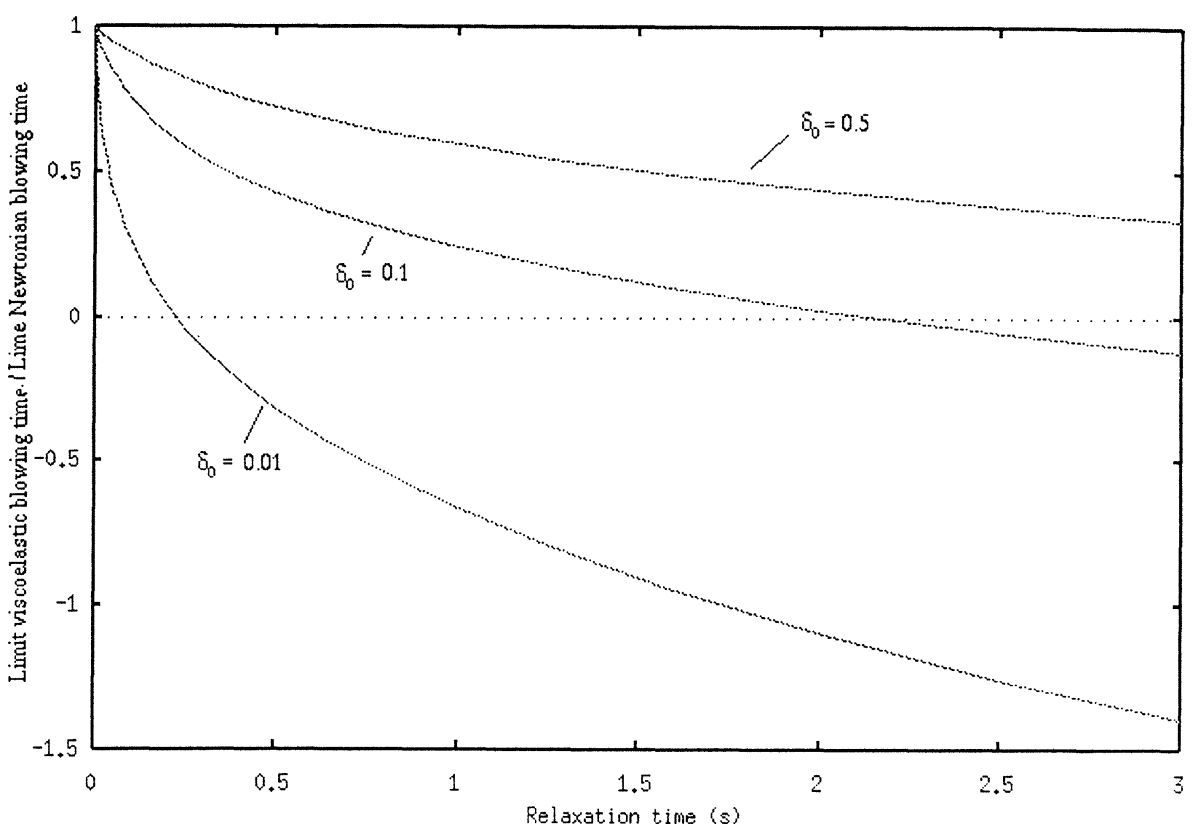

Figure 7. Limit blowing times of viscoelastic membranes.

It is to notice that for the viscoelastic volumic approach, this limit blowing times is equivalent to be thin shell one (see figure 5) when convergence may be obtained.

These analytical models provide useful reference solutions for both volumic and thin shell finite element numerical models.

In addition, comparisons between the analytical and finite element simulations allows us to validate the numerical models.

\section{References}

Agassant J.-F., Avenas P., Sergent J.-Ph., Carreau P.J., 1991. Polymer Processing_Principles and Modeling. Ed. Carl Hansen, New York.

Agassant J.-F., Avenas P., Sergent J.-Ph., Vergnes B., 1996. La mise en forme des matières plastiques. Technique et Documentation, Paris.

Chung S.C.K., Stevenson J.F., 1975. A general elongation experiment: inflation and extension of a viscoelastic tube. Rheo. Act. 14, 832-841.

Dutta A., Ryan M.E., 1984. Confined parison inflation behavior of a high-density polyéthylène. Polym. Eng. Sci. 24, 1232-1239.

Khayat R.E., Garcia-Rejon A., 1992. Uniaxial and biaxial unsteady inflations of a viscoelastic material. J. Non New. Fluid Mech. 43, 31-59.

Lodge A.S., 1964. Elastic Liquids, Academic Press, New York.

Rodriquez-Villa A., 1997. Etude théorique et expérimentale de l'extrusion-soufflage de corps creux en polymères. Ph.D. thesis (in French), Ecole des Mines de Paris.

Rosato D.V., 1989. Blow Molding Handbook. Carl Hahsen, New York.

Ryan M.E., Dutta A., 1982. The dynamics of parison free inflation in extrusion blow molding. Polym. Eng. Sci. 22, 569-577.

Schmidt F.M., 1995. Etude experimentale et modélisation du procédé d'injection-souflage bi-orientation en cycle froid de bouteilles en PET. Ph.D. thesis (in French), Ecole des Mines de Paris.

Schmidt F.M., Agassant J.F., Bellet M., Desoutter L., 1996. Viscoelastic simulation of P.E.T. stretch/blow molding. J. Non Newt. Fluid 64, $19-42$. 